\title{
La rénovation urbaine, une opportunité de réduire les inégalités socio-spatiales d'être impliqué dans un accident dans les espaces publics
}

\section{Sylvanie Godillon}

\section{(2) OpenEdition Journals}

Édition électronique

URL : http://journals.openedition.org/cdg/1035

DOI : $10.4000 /$ cdg. 1035

ISSN : 2107-7266

Éditeur

UMR 245 - CESSMA

Référence électronique

Sylvanie Godillon, "La rénovation urbaine, une opportunité de réduire les inégalités socio-spatiales d'être impliqué dans un accident dans les espaces publics ", Carnets de géographes [En ligne], 4 | 2012, mis en ligne le 01 septembre 2012, consulté le 24 septembre 2020. URL : http:// journals.openedition.org/cdg/1035; DOI : https://doi.org/10.4000/cdg.1035

\section{(c) (†) $९$}

La revue Carnets de géographes est mise à disposition selon les termes de la Licence Creative Commons Attribution - Pas d'Utilisation Commerciale - Pas de Modification 4.0 International. 


\title{
La rénovation urbaine, une opportunité de réduire les inégalités socio-spatiales d'être impliqué dans un accident dans les espaces publics
}

\author{
SYLVANIE GODILLON
}

Si l'insécurité routière a globalement diminué depuis les années 1970 avec une nette amélioration depuis 2002, les différentes catégories de population ne sont pas égales devant le risque d'accident. De récents travaux de recherche montrent que les habitants des quartiers défavorisés ont plus d'accidents que des habitants de quartiers aisés (Edwards et al., 2006 ; Fleury et al., 2009). Dans la mesure ou les personnes pauvres ont 20 à $40 \%$ de plus d'accidents que les personnes aisées (Hasselberg et al., 2001), un effet de composition peut expliquer les risques supplémentaires. Autrement dit les habitants des quartiers défavorisés auraient plus d'accidents en raison de leur profil social. Des travaux récents décomposent l'influence des différentes variables individuelles, familiales et sociales de celles liées aux contextes socio-économiques du quartier de résidence et montrent que les risques d'accidents sont plus forts pour les habitants de quartiers pauvres (Cubbin et al., 2000 ; O'Campo et al., 2000 ; Simpson et al., 2005).

La concentration de la pauvreté n'est pas le seul facteur explicatif : la forme urbaine du quartier de résidence joue également un rôle. Mueller et al. montrent que les enfants qui résident dans des immeubles ont 5,5 fois plus de risques d'accident que les enfants habitant des maisons individuelles (Mueller et al., 1990) en raison d'une exposition plus grande au risque avec des temps importants passés en dehors du logement. De plus, la forme urbaine du quartier influence les représentations spatiales des individus puisqu'un réseau viaire complet et hiérarchisé facilite l'apprentissage de l'espace (Faure, Garnier, 1994).

Effet de composition, effet de la concentration de la pauvreté, effet de la forme urbaine du quartier... quels sont les mécanismes explicatifs de ces différentes influences sur le risque individuel ? Ces analyses interpellent les actions publiques d'une géographie prioritaire qui cible les quartiers défavorisés. Un des objectifs du Programme National de Rénovation Urbaine est de désenclaver ces quartiers pour réduire l'insécurité civile et ainsi apaiser la cohabitation entre les habitants. Dans ce cadre, la rénovation urbaine est-elle une opportunité à saisir pour réduire les inégalités devant les risques d'accidents?

L'influence de la forme urbaine sur le risque des habitants est essentielle à comprendre dans un contexte de rénovation urbaine. Depuis 2003, le Programme National de Rénovation Urbaine a pour objectif de transformer les quartiers pauvres à travers notamment des actions sur la forme du bâti. Intégrer les enjeux de sécurité routière dans 
les processus de décision des projets de rénovation urbaine relève des questions de coordination entre politiques sectorielles.

Pour comprendre ces processus, les risques des habitants sont étudiés à partir de neuf quartiers défavorisés de l'agglomération lilloise. Ces risques sont mis en relation avec les caractéristiques sociales, urbaines et accidentologiques de leur quartier par un Système d'Information Géographique (SIG). Cette thèse est novatrice dans la mesure où elle utilise et croise des matériaux originaux. La constitution d'une base de données à partir des procès-verbaux d'accidents qui renseignent les caractéristiques sociales et spatiales des individus est croisée avec des informations relatives à la mobilité et aux caractéristiques de l'espace.

La combinaison de méthodes statistiques, épidémiologiques et des analyses spatiales permettent d'identifier des processus de production des inégalités devant les risques d'accidents. Des analyses écologiques multiniveaux et spatiales montrent que la pauvreté du lieu de résidence accentue les risques de catégories déjà plus impliquées (hommes, jeunes, professions intermédiaires) et détermine fortement le risque d'avoir un accident en tant que piéton. Une suroccupation des logements dans le quartier de résidence, associée à une forte densité de réseau et à de forts trafics, augmente significativement les risques. En étudiant les processus de production des inégalités socio-spatiales devant les risques d'accidents lors des déplacements, la thèse cible les aspects socio-économiques de la mobilité. Après avoir analysé ces processus et modélisé les répartitions spatiales des accidents, la possibilité de réduire ces inégalités par la rénovation urbaine est appréhendée.

Les influences du contexte résidentiel interrogent les actions du Programme National de Rénovation Urbaine. L'intérêt du travail a été de s'interroger sur la prise en compte des enjeux de sécurité des transports dans la planification des projets urbains financés en partie par l'Agence Nationale pour la Rénovation Urbaine. Les quartiers défavorisés bénéficient en effet de programmes urbains et sociaux dont les objectifs sont de réduire les inégalités et d'améliorer la qualité du cadre de vie. Si les inégalités de risques des habitants ne sont pas connues, les situations d'insécurité dans leur quartier de résidence sont-elles traitées par ces programmes ? La prise en compte des enjeux de sécurité par les projets de rénovation urbaine pose la question de la coordination entre différentes politiques sectorielles. Une démarche comparative permet de saisir les spécificités françaises à travers des éclairages allemand et britannique. L'Allemagne et le RoyaumeUni ont en effet deux façons très différentes de coordonner les politiques sectorielles. Les autorités locales sont plus autonomes en Allemagne qu'en France, ce qui facilite une meilleure prise en compte des spécificités locales (Blanc, 2006). Les processus de décision sont menés en associant au plus tôt l'ensemble des acteurs en lien avec le sujet à travers le concept de développement intégré. Cette intégration des différents acteurs en amont des projets urbains laisse supposer une meilleure connaissance des spécificités d'insécurité dans les quartiers. Au contraire, le Royaume-Uni est très centralisé et fonctionne par des financements dédiés (Menerault et al., 2007). En effet, le gouvernement britannique a mis en place en 2002 un fonds pour réduire le nombre de tués et de blessés graves dans les quartiers défavorisés : la Neighbourhood Road Safety Initiative (NRSI). L'étude de ce procédé permet de constater l'efficacité d'un financement dédié a un objectif précis par rapport à des processus de décision plus intégrés. Les résultats permettent de proposer des solutions innovantes pour une 
meilleure coordination des secteurs de la rénovation urbaine et des opérations de transport dans le cadre de la rénovation urbaine. Une comparaison à plusieurs échelles de la France (Lille Métropole Communauté Urbaine) avec l'Allemagne (Dresde) et le Royaume-Uni (Grand Manchester) montre que les projets français favorisent les fonctions de circulation sans prendre en considération les effets sur l'insécurité. Ce programme gagnerait donc à intégrer les enjeux de sécurité des déplacements afin de réduire ces inégalités socio-spatiales.

\section{Références}

BLANC, M., 2006, "Politique de la ville et Soziale Stadt, une comparaison francoallemande », Pensées Plurielles, Vol. 12, n 2, p. 45-51.

CUBBIN, C., LECLERE, F.B., SMITH, G.S., 2000, « Socioeconomic status and injury mortality: individual and neighbourhood determinants », Journal of Epidemiology and Community Health, Vol. 54, n 7, p. 517-524.

DWARDS, P., GREEN, J., ROBERTS, I., GRUNDY, C., LACHOWYCS, K., 2006, Deprivation and road safety in London : A report to the London Road Safety Unit, London, London School of Hygiene \& Tropical Medicine.

FAURE, A., GARNIER, F., 1994, Les jeunes, les grands ensembles et la sécurité routière, Paris, Rapport de Arch'urba pour la DRAST (Direction de la Recherche et des Affaires Scientifiques et Techniques).

FLEURY, D., PEYTAVIN, J.-F., ALAM, T., GODILLON, S., SAINT-GERAND, T., MEDJKANE, M., BLONDEL, C., BENSAID, K., MILLOT, M., 2009, Disparité des Espaces du Risque Routier, Salon de Provence, Rapport de recherche de l'INRETS.

HASSELBERG, M., LAFLAMME, L., RINGBACK WEITOFBT, G., 2001, "Socioeconomic differences in road traffic injuries during childhood and youth : a closer look at different kinds of road user », Journal of Epidemiology and Community Health, Vol. 55, p. 858-862.

MENERAULT, P., L'HOSTIS, A., HARMAN, R., 2007, «Les transports urbains et régionaux face à un avenir incertain », dans BOOTH, P., BREUILLARD, P., FRASER, C., PARIS, D. (sous la dir.), Aménagement et urbanisme en France et en GrandeBretagne - Étude comparative, Paris, L'Harmattan, p. 297-320.

MUELLER, B.A., RIVARA, F.P., LII, S.M., 1990, «Environmental factors and the risk for childhood pedestrian-motor vehicle collision occurrence», American Journal of Epidemiology, Vol. 132, p. 550-560.

O'CAMPO, P., RAO, R., GIELEN, A.-C., ROYALTY, W., WILSON, M., 2000, « Injury- producing events among children inb low-income communities: the role of community caracteristics », Journal of Urban Health : Bulletin of the New-York Academy of Medecine, Vol. 77, $\mathrm{n}^{\circ}$ 1, p. 34-39.

SIMPSON, K., JANSSEN, I., CRAIG, W.M., PICKETT, W., 2005, « Multilevel analysis of associations between socioeconomic status and injury among Canadian adolescents », Journal of Epidemiology and Community Health, Vol. 59, n 12, p. 10721077. 


\section{Fiche informative}

\section{Discipline}

Géographie-Aménagement

Directeurs de thèse :

Francis Beaucire

Dominique Fleury

\section{Université}

Université Paris 1 Panthéon-Sorbonne

Membres du jury de thèse, soutenue le 27 mars 2012

Francis BEAUCIRE, Professeur, Université Paris 1 - Panthéon-Sorbonne (directeur) Gabriel DUPUY, Professeur, Université Paris 1 - Panthéon-Sorbonne Dominique FLEURY, Directeur de recherche, IFSTTAR (directeur) Sylvie FOL, Professeur, Université Paris 1 - Panthéon-Sorbonne Vincent KAUFMANN, Professeur, Université Polytechnique de Lausanne, (rapporteur) Maurizio TIRA, Professeur, Université de Brescia (rapporteur)

\section{Situation professionnelle actuelle}

Chargée d'études et de recherche à 6T-Bureau de recherche

Contact

godillon_sylvanie@yahoo.fr 